\title{
MIN-MAX OPTIMIZATION OF EMERGENCY SERVICE SYSTEM BY EXPOSING CONSTRAINTS
}

This paper deals with the fair public service system design using the weighted p-median problem formulation. Studied generalized system disutility follows the idea that the individual user's disutility comes from more than one located service center and the contributions from relevant centers are weighted by some coefficients. To achieve fairness in such systems, various schemes may be applied. The strongest criterion consists in the process when the disutility of the worst situated users is minimized first, and then the disutility of better located users is optimized under the condition that the disutility of the worst situated users does not worsen. Hereby, we focus on the first step and try to find an effective solving method based on the radial formulation. The main goal of this study is to show how suitable solving method for the min-max optimal system design can save computational time and bring precise results.

Keywords: Public service system, generalized disutility, radial min-max approach.

\section{Introduction}

The public service system design problem is a challenging task for both system designer and operational researcher. As the first one searches for a tool which enables to obtain service center deployment satisfying future demands of system users, the second one faces the necessity of completing the associated solving tool. The family of public service systems includes medical emergency system, public administration system and many others where the quality criterion of the design takes into account some evaluation of users' discomfort [1], [2], [3] and [4]. Thus designing of a public service system includes determination of limited number of center locations from which the service is distributed to all users. The associated objective in the standard formulation is to minimize some form of disutility which is proportional to the distance between served users and the nearest service centers [5] and [6]. This paper is focused on such methods of the public service system design where the generalized disutility is considered instead of common distance. It follows the idea of random occurrence of the demand for service and limited capacity of the service centers in real emergency rescue systems [6] and [7]. At the time of the current demand for service, the nearest service center may be occupied by some other user for which this service center is also the nearest one. When such situation occurs, the current demand is usually served from the second nearest center or from the third nearest center if the second one is also occupied. Thus we assume that the service is generally provided from more located service centers and the individual contributions from relevant centers may be weighted by reduction coefficients depending on a center order [6] and [8]. This approach constitutes an extension of previously developed methods where only one nearest center was taken as a source of individual user's disutility. Furthermore, we pay attention to the quality criterion of the design. On the contrary to our previous research where the average user's disutility was minimized, here we focus on the fair-optimal public service system design.

Fairness in general emerges whenever limited resources are to be fairly distributed among participants [9], [10], [11] and [12]. The strongest scheme is so-called lexicographic min-max criterion. Applying this scheme, the disutility perceived by the worst situated user is minimized first and then the disutility of the second worst situated user is minimized unless the previously achieved disutility of the worst situated users is worsened. This approach is applied step by step for the remaining users [13].

Complexity of previously described problems has led to searching for a suitable algorithm which complies with the task. It was found that the radial formulation of the problem can considerably accelerate the associated solving process [14]. Simultaneously with the above mentioned research, an attention was paid to so-called approximate approaches which make use of commercial IP-solvers and the radial formulation with homogenous system of radii concerning individual users [15] and

\footnotetext{
* ${ }^{1}$ Jaroslav Janacek, ${ }^{2}$ Marek Kvet

${ }^{1}$ Faculty of Management Science and Informatics, University of Zilina, Slovakia

${ }^{2}$ University Science Park, University of Zilina, Slovakia

E-mail: jaroslav.janacek@fri.uniza.sk
} 
[16]. These approaches are called approximate not due to the solving tool, but for some small impreciseness connected with rounding the disutility values up to values from the set of so-called dividing points.

In this paper, we focus on the first step of the lexicographic approach which consists in solving the min-max optimal public service system design problem where the disutility of the worst situated user is minimized. We study and compare two different approaches from the point of their impact on the solution accuracy and saved computational time.

The remainder of the paper is organized as follows. Section 2 introduces the generalized model of individual user's disutility concerning more than one contributing center and provides the mathematical formulation of the problem based on the radial formulation. Section 3 contains the description of suggested approximate bisection search for exposing structure and gives the resulting algorithm for the min-max location problem solution. Section 4 contains numerical experiments, comparison of the suggested approaches and Sections 5 gives final conclusions.

\section{Generalized disutility and min-max criterion in public service system}

\subsection{Generalized system disutility}

To formulate a mathematical model of the min-max optimal public service system design problem, we denote the set of user locations by $J$ and the set of possible service center locations will be denoted by $I$. The basic decisions in any solving process of the problem concern location of given number $p$ of centers at the possible locations from the set $I$. The system disutility for the user located at $j \in J$ provided by a center located at $i \in I$ is denoted by $d_{i j}$. The randomly restricted capacity of a service center can be generalized so that the $r$ nearest located centers influence the total disutility perceived by any user. In this paper, the generalized disutility for any user is modeled by a sum of weighted disutility contributions from the $r$ nearest centers. The weights $q_{k}$ for $k=1$ $\ldots r$ are positive real values which meet the following inequalities $q_{1} \geq q_{2} \geq \ldots \geq q_{\dot{r}}$. The $k$-th weight can be proportional to the probability of the case that the $k-1$ nearest located centers are occupied and the $k$-th nearest center is available [17].

\subsection{Radial formulation}

We assume that the disutility contribution value ranges only over non-negative integers from the range $\left[d^{0}, d^{m}\right]$ of all possible disutility values $d^{0}<d^{l}<\ldots<d^{m}$ from the matrix $\left\{d_{i j}\right\}$. The values partition the range into $m=v+1$ zones. The zone $s$ corresponds with the interval $\left(d^{s}, d^{s+l}\right]$. The length of the $s$-th interval is denoted by $e_{s}$ for $s=0 \ldots v$. To describe the system of radii formed by the values [8], a system of zero- one constants is defined so that the constant $a_{i j}^{s}$ is equal to 1 if and only if the disutility contribution $d_{i j}$ for a user from location $j$ from the possible center location $i$ is less or equal to $d^{s}$, otherwise $a_{i j}^{s}$ is equal to 0 . Let the location variable $y_{i} \in\{0,1\}$ model the decision of service center location at the location $i \in I$ by the value of 1 . Further, we introduce auxiliary zero-one variables $x_{j s k}$ for $j \in J, s \in[0 \ldots v], k \in[1 \ldots r]$ to model the disutility contribution value of the $k$-th nearest service center to the user $j$. The variable $x_{j s k}$ takes the value of 1 if the $k$-th smallest disutility contribution for the customer $j \in J$ is greater than $d^{s}$ and it takes the value of 0 otherwise. Then the expression $e_{0} x_{j 0 k}+e_{T} x_{j l k}+e_{2} x_{j 2 k}+\ldots+e_{v j} x_{j k k}$ constitutes the $k$-th smallest disutility contribution $d_{j^{*}}^{k}$ for customer located at $j$. Under mentioned preconditions, we can describe the min-max optimal public service system design problem using the following variables and other denotations.

\section{Minimize $h$}

Subject to $\sum_{i \in I} y_{i} \leq p$

$\sum_{k=1}^{r} x_{j s k}+\sum_{i \in I} a_{i j}^{s} y_{i} \geq r$ for $j \in J, s=0, \ldots, v$

$\sum_{k=1}^{r} q_{k} \sum_{s=0}^{r} e_{s} x_{j s k} \leq h$ for $j \in J$

$y \in\{0,1\}$ for $i \in I$

$x_{j s k} \in\{0,1\}$ for $j \in J, s=0, \ldots, v, k=1, \ldots, r$

$h \geq 0$

The constraint (2) puts a limit $p$ on the number of located centers. The constraints (3) ensure that the sum of variables $x_{j s k}$ over $k \in[1 \ldots r]$ expresses the number of the service centers outside the radius $s$ from the user location $j$, which remains to the number $r$. The link-up constraints (4) ensure that each perceived disutility is less than or equal to the upper bound $h$. Validity of the assertion that the expression on the left-hand-side of (4) expresses the sum $q_{I} d_{i l, j}+q_{2} d_{i 2, j}+\ldots+q_{r} d_{i r, j}$ of weighted relevant disutility values from the $r$ nearest service centers $i 1, i 2, \ldots$, ir to the user located at $j$ follows from the next reasoning. It can be easily found that minimal sum of the variables $x_{i s k}$ over $k \in[1 \ldots r]$ completes the number of located service centers in the radius $s$ from user location $j$ to the number $r$. This way, the sum gives the number $t$ of the nearest service centers whose disutility contribution is greater than or equal to the value $d^{s}$. As the sequence of $q_{k}$ decreases, only $x_{j s k}$ for $k=r-t+1, r-t+2 \ldots r$ must be equal to one for the given $j$ and $s$. It causes that the biggest disutility contribution is assigned by the smallest value of $q_{k}$. The left-hand-side of (4) is pushed down by some optimization process and then the constraints $x_{j s k} \leq x_{j s-1, k}$ for $s=1 \ldots v$ must hold due to construction of $a_{i j}^{s}$ and constraints (3) and further also the constraints $x_{j s k} \leq x_{j k k+1}$ for $k=1 \ldots r-1$ must hold due to convexity given by decreasing sequence of $q_{k}$. 


\section{The approximate bisection search for exposing structure}

\subsection{Exposing structure for the radial formulation}

Let us consider the radial formulation (1) - (7) of the generalized $p$-center problem with the zero-one coefficients $a_{i j}^{s}$ defined for $i \in I, j \in J$ and $s \in[0 \ldots v]$ where $r$ nearest located centers influence the disutility perceived by a user. The coefficients are derived from the disutility contribution values which range only over non-negative integers of all possible disutility values $d^{0}<d^{l}<\ldots<d^{m}$ from the matrix $\left\{d_{i j}\right]$. The triple $[u$, $S, G]$ is denoted as an exposing structure, if its components satisfy the following rules. The first component $u$ is a positive integer less than or equal to $r$. The second component $S$ is an $u$-tuple $[S(1), \ldots$, $S(u)$ ], of nonnegative increasing integers where $0 \leq S(1)<S(2)<$ $\ldots<S(u) \leq m$. The third component is $u$-tuple $[G(1), \ldots, G(u)]$ of positive increasing integers where $1 \leq G(1)<G(2)<\ldots<G(u) \leq$ $r$. If $G(u)=r$, then the structure is denoted as complete structure. Using the above introduced location variables $y_{i} \in\{0,1\}$ for $i \in I$, the following set of constraints can be formulated for the exposing structure $[u, S, G]$

$\sum_{i \in I} y_{i} \leq p$

$\sum_{i \in I} a_{i j}^{S(w)} y_{i} \geq G(w)$ for $j \in J, w \in[1 . . u]$

$y_{i} \in\{0,1\}$ for $i \in I$

If a feasible solution $\boldsymbol{y}$ of the constraints (8) - (10) structure exists for a complete $[u, S, G]$, then each user location $j$ must lie at least in the radius $d^{S(l)}$ from $G(1)$ located service centers and in the radius $d^{S(2)}$ from $G(2)-G(1)$ additional service centers and so on till up to the radius $d^{S(u)}$ from the $G(u)-G(u-1)$ service centers. It means that the worst situated user perceives the generalized disutility less than or equal to the value of (11)

$H_{[u, S, G]}=d^{S(1)} \sum_{k=1}^{G(1)} q_{k}+\sum_{w=2}^{u} d^{S(w)} \sum_{k=G(w-1)+1}^{G(w)} q_{k}$

An exposing structure is called valid if there is at least one feasible solution of the problem (8) - (10) formulated for the structure.

\subsection{Ordering on the set of complete exposing structures}

Let us introduce a mapping $E_{[u, S, G \mid}[1 . . r] \AA\{S(1), \ldots, S(u)\}$ for each complete structure $[u, S, G]$, to be able to characterize the properties of complete structures more easily. The mapping $E_{|u S G|}$

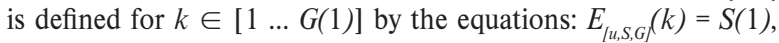
and for $k \in[G(w-1)+1 . . G(w)]$ by the equations: $E_{[u, S, G]}(k)=S(w)$ where $w=2 \ldots u$.

\section{Definition}

We say that the complete structure $[u, S, G]$ dominates the complete structure $[\underline{u}, \underline{S}, \underline{G}]$ if the inequality $E_{[u, S, G]}(k) \leq E_{[\underline{\underline{u}, \underline{G} G \mid}}(k)$ holds for each $k$.

\section{Proposition 1}

If a complete structure $[u, S, G]$ dominates another complete structure $[\underline{u}, \underline{S}, \underline{G}]$, then an subscript $w^{*}(\underline{w}) \in[1 . . u]$ must exist for any $\underline{w} \in[1 . . \underline{u}]$ such that $G\left(w^{*}(\underline{w})\right) \geq \underline{G}(\underline{w})$ and $S\left(w^{*}(\underline{w})\right) \leq \underline{S}(\underline{w})$.

Proof: We perform the proof by contradiction assuming that there exists a subscript $\underline{w}$ such that either $G(w)<\underline{G}(\underline{w})$ or $S(w)>\underline{S}(\underline{w})$ holds for each $w \in[1 . . u]$. As $S(w)<S(w+1)$ and $G(w)<G(w+1)$ follow from definition of the exposing structure and $S(1) \leq \underline{S}(1)$ follows the structure domination, then maximal $w^{\prime}$ exists such that $S\left(w^{\prime}\right) \leq \underline{S}(\underline{w})$ and $G\left(w^{\prime}\right)<\underline{G}(\underline{w})$. It follows that the range $\left[G\left(w^{\prime}\right)+1 . . \underline{G}(\underline{w})\right]$ of integers is nonempty and each element $l$ of this range satisfies the inequalities $E_{|u, S, G|}(l) \leq \underline{S}(\underline{w})$ and

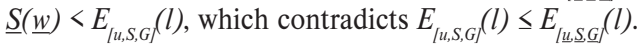

\section{Proposition 2}

Let us denote $Y$ and $\underline{Y}$ sets of all feasible solutions satisfying constraints (8)-(10) for complete structures $[u, S, G]$ and $[\underline{u}, \underline{S}$, $\underline{G}]$ respectively. If the structure $[u, S, G]$ dominates the structure $[\underline{u}, \underline{S}, \underline{G}]$, then $Y \subseteq \underline{Y}$ holds.

Proof: Let us notice that only constraints (9) are influenced by the considered structures and that $s^{\prime} \geq s$ implies the inequality $a_{i j}^{s^{\prime}} \geq a_{i j^{\prime}}^{s}$ Considering a solution $\boldsymbol{y} \in Y$, the constraint (9) according to $[u, S, G]$ is satisfied for each $j \in J$ and $w \in[1 . . u]$. According to Proposition 1, subscript $w^{*}(\underline{w}) \in[1 . . u]$ must exist for any $\underline{w} \in[1 . . \underline{u}]$ such that $G\left(w^{*}(\underline{w})\right) \geq \underline{G}(\underline{w})$ and $S\left(w^{*}(\underline{w})\right) \leq \underline{S}(\underline{w})$. Then the inequalities (12) hold for each $j \in J$ and $\underline{w} \in[1 . . \underline{u}]$.

$\underline{G}(\underline{w}) \leq G\left(w^{*}(\underline{w})\right) \leq \sum_{i \in I} a_{i j}^{S\left(w^{*}(\underline{w})\right)} y_{i} \leq \sum_{i \in I} a_{i j}^{S(\underline{w})} y_{i}$

From the inequalities (12) it follows that $y$ also satisfies (9) according to $[\underline{u}, \underline{S}, \underline{G}]$.

Proposition 3

If the structure $[u, S, G]$ dominates the structure $[\underline{u}, \underline{S}, \underline{G}]$, then $H_{[u, S, G]} \leq H_{[u, \underline{S}, \underline{G}]}$ holds.

Proof: Note that equalities (13) and (14) hold.

$$
\begin{aligned}
& H_{[u, S, G]}=d^{S(1)} \sum_{k=1}^{G(1)} q_{k}+\sum_{w=2}^{u} d^{S(w)} \sum_{k=G(w-1)+1}^{G(w)} q_{k}= \\
& \sum_{k=1}^{G(1)} d^{E[u, G] \mid k)} q_{k}+\sum_{w=2}^{u} \sum_{k=G(w-1)+1}^{G(w)} d^{E[u, S, G](k)} q_{k}= \\
& \sum_{k=1}^{u} d^{E[\mu, S, G](k)} q_{k} \\
& H_{[\underline{u}, \underline{S}, \underline{G}]}=d^{\underline{S}} \underline{\underline{S}(1)} \sum_{k=1}^{G(1)} q_{k}+\sum_{w=2}^{\underline{u}} d^{\underline{S}(w)} \sum_{k=\underline{G}(w-1)+1}^{\underline{G}(w)} q_{k}= \\
& \sum_{k=1}^{u} d^{E[\underline{L}, \underline{S} \underline{G}](k)} q_{k}
\end{aligned}
$$


As $d^{E[u, S, G /(k)} \leq d^{E / u}, \underline{S} \underline{G} /(k)$ holds for each $k \in[1 . . r]$, then $H_{[u, S,}$ ${ }_{G]} \leq H_{[\underline{\underline{L}}, \underline{G}, \underline{G}}$ also holds.

\section{Proposition 4}

The ordering defined on the set of all complete exposing structures by relation of dominance is not complete ordering in general.

Proof: The proof is performed by the construction of two examples where each of them includes two complete structures. None of the structures dominates the other and, furthermore, there is no further structure which dominates any of the two ones. In addition, the pair of examples shows that mutual positions of the lowest subscripts $S(1)$ and $\underline{S}(1)$ do not decide which structure takes the lowest value of (11).

Both of the following examples are defined on the network where the set $J$ of users' locations contains only two elements $J=\{1,2\}$ and the set of possible center locations $I$ consists of four elements $I=\{1,2,3,4\}$. It is necessary to locate $p=2$ centers so that the generalized disutility for the worst situated user is minimal The generalized disutility is defined here for $r=2$ and for reduction coefficients $q_{1}=1$ and $q_{2}=0.5$.

\section{Example 1}

This example is defined for the matrix of disutility contributions $d_{i j}$ in Table 1 and for the associated sequence of the different values $d^{0}<d^{l}<\ldots<d^{m}$ from the matrix $\left\{d_{i j}\right]$ in the form $1<4<9$. The associate matrices of $a_{i j}^{s}$ for $s=0,1,2$ are depicted in Table 2.

Potential disutility contributions $d_{i j}$ from the center locations to the user locations

Table 1

\begin{tabular}{|c|l|l|}
\hline$d_{i j}$ & 1 & 2 \\
\hline 1 & 1 & 9 \\
\hline 2 & 4 & 4 \\
\hline 3 & 4 & 4 \\
\hline 4 & 9 & 1 \\
\hline
\end{tabular}

Matrices of $a_{i j}^{s}$ for $s=0,1,2$

Table 2

\begin{tabular}{|c|c|c|c|c|c|c|}
\hline$s:$ & \multicolumn{2}{|c|}{0} & \multicolumn{2}{|c|}{1} & \multicolumn{2}{|c|}{2} \\
\hline$i \mid j$ & 1 & 2 & 1 & 2 & 1 & 2 \\
\hline 1 & 1 & 0 & 1 & 0 & 1 & 1 \\
\hline 2 & 0 & 0 & 1 & 1 & 1 & 1 \\
\hline 3 & 0 & 0 & 1 & 1 & 1 & 1 \\
\hline 4 & 0 & 1 & 0 & 1 & 1 & 1 \\
\hline
\end{tabular}

Let us define two complete exposing structures $[u, S, G]=$ $[2,[0,2],[1,2]]$ and $[\underline{u}, \underline{S}, \underline{G}]=[1,[1],[2]]$ for this example. We obtain $H_{[u, S, G]}=5.5$, mapping $E_{[u, S, G]}(1)=0, E_{[u, S, G}(2)=2$, and we can easily find that there is only one feasible solution $\boldsymbol{y}=\left[y_{p}, y_{2}, y_{3}, y_{4}\right]=[1,0,0,1]$ of (8)-(9) for the structure $[u, S$, $G]=[2,[0,2],[1,2]]$. Similarly, we obtain $H_{[\underline{\underline{S}} \underline{\underline{S}}, \underline{G}]}=6$ mapping

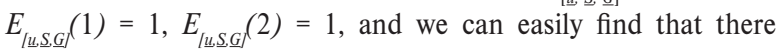
is only one feasible solution $\boldsymbol{y}=\left[y_{1}, y_{2}, y_{3}, y_{4}\right]=[0,1,1,0]$ of (8)-(9) for the structure $[\underline{u}, \underline{S}, \underline{G}]=[1,[1],[2]]$. It can be easily found that none of the structures dominates the other and that $H_{[u, S, G]}<H_{[\underline{[u}, \underline{G}, \underline{G}]}$ subject to $S(1) \underline{\underline{S}}(1)$.

\section{Example 2}

This example is defined for the matrix of disutility contributions $d_{i j}$ in Table 3 and for the associated sequence of the different values $d^{0}<d^{l}<\ldots<d^{m}$ from the matrix $\left\{d_{i j}\right\}$ in the form $1<2<5$. The associate matrices of $a_{i j}^{s}$ for $s=0,1,2$ are depicted in Table 4.

Potential disutility contributions $d_{i j}$ from the center locations to the user locations.

Table 3

\begin{tabular}{|c|l|l|}
\hline$d_{i j}$ & 1 & 2 \\
\hline 1 & 1 & 5 \\
\hline 2 & 2 & 2 \\
\hline 3 & 2 & 2 \\
\hline 4 & 5 & 1 \\
\hline
\end{tabular}

Matrices of $a_{i j}^{s}$ for $s=0,1,2$.

Table 4

\begin{tabular}{|c|c|c|c|c|c|c|}
\hline$s:$ & \multicolumn{2}{|c|}{0} & \multicolumn{2}{|c|}{1} & \multicolumn{2}{|c|}{2} \\
\hline$i \mid j$ & 1 & 2 & 1 & 2 & 1 & 2 \\
\hline 1 & 1 & 0 & 1 & 0 & 1 & 1 \\
\hline 2 & 0 & 0 & 1 & 1 & 1 & 1 \\
\hline 3 & 0 & 0 & 1 & 1 & 1 & 1 \\
\hline 4 & 0 & 1 & 0 & 1 & 1 & 1 \\
\hline
\end{tabular}

Let us define two complete exposing structures $[u, S, G]=[2$, $[0,2],[1,2]]$ and $[\underline{u}, \underline{S}, \underline{G}]=[1,[1],[2]]$ for this example. We obtain $H_{[u, S, G]}=3.5$, mapping $E_{[u, S, G\}}(1)=0, E_{\langle u, S, G}(2)=2$, and we can easily find that there is only one feasible solution $\boldsymbol{y}=\left[y_{p}, y_{2}, y_{3}, y_{4}\right]=$ $[1,0,0,1]$ of (8)-(9) for the structure $[u, S, G]=[2,[0,2],[1,2]]$. Similarly we obtain $H_{[\underline{\underline{S}} \underline{\underline{S}}, \underline{G}]}=3$ mapping $E_{[\underline{\underline{u}, \underline{G} G]}}(1)=1, E_{[\underline{u} \underline{S} \underline{G}]}(2)=1$, and we can easily find that there is only one feasible solution $\boldsymbol{y}=\left[y_{p}, y_{2}, y_{3}, y_{4}\right]=[0,1,1,0]$ of (8)-(9) for the structure $[\underline{u}, \underline{S}, \underline{G}]$ $=[1,[1],[2]]$. It can be easily found that none of the structures dominates the other and that $H_{[u, S, G]}>H_{[u, \underline{S} \underline{G}]}$ subject to $S(1)<\underline{S}(1)$.

\subsection{Lexicographic maximal completion of valid exposing structure}

The process of valid structure completion starts with an incomplete valid structure $[u, S, G]$ where $G(u)<r$. As we assume that the structure is valid, at least one feasible solution of (8) - (11) exists. The lexicographic process begins with attempt 
to increase the value of $G(u)$ as much as possible keeping the augmented structure valid. As the possible increase of $G(u)$ is limited by $r$, only finite and small number of tests of solution existence is necessary to determine the highest value of $G(u)$. The associated algorithm will be called "AugmentG".

Further, let us consider incomplete structure which has been processed by the algorithm AugmentG. The lexicographical process continues with attempt to add next subscript $S(u+1)$ to the structure as the lowest subscript from the range $[S(u)+1 . . m]$. The associated value of $G(u+1)$ is set at the lowest possible value, i.e. $G(u)+1$. The searching process can be finished quickly even for big value of $m$ when bisection is used. If the value of $m$ is used as upper bound of the searched subscript, the algorithm ever succeeds in the search. If the searched range is reduced, then addition may fail. The associated algorithm will be called "AugmentS".

After successful run of the algorithm, the structure is enlarged by one element of the array $S$ and $G$ and $u$ is increased by one.

The process of the structure completion can be performed by the following steps.

Step 0 . Initialize the starting valid incomplete structure $[u, S, G]$. [Comment: $G(u)<r$ holds for the incomplete structure.]

Step 1. Repeat the following two steps until $G(u)=r$.

Step 2. Apply AugmentG on $[u, S, G]$.

[Comment: The previous step may increase $G(u)$ so that structure stay valid.]

Step 3. If $G(u)<r$, apply AugmentS on $[u, S, G]$.

[Comment: The previous step increases $u$ to $u+1$, adds elements $S(u+1)$ and $G(u+1)$ to the $u$-tuples $S$ and $G$ respectively so that the augmented structure stays valid and $S(u+1)>S(u)$ and $G(u+1)=$ $G(u)+1$.

If the search included in the algorithm Augments is performed over the range $[S(u)+1 . . m]$, then the resulting structure of-is a complete valid exposing structure and in addition, any other valid structure containing the starting structure cannot dominate the resulting complete structure. If the search is restricted on some smaller range, e.g. not to produce complete structures with the value $H_{[u, S, G]}$ higher than a given upper bound $U B$, then the process can be prematurely stopped unless valid complete structure is produced. The above process will be called "Complete" in the remainder of the paper.

\subsection{An approximate algorithm for min-max location problem solution}

The suggested approximate algorithm is based on the partial search over set of non-dominated complete valid exposing structures where inspected structures are obtained by the lexicographic maximal completion of "seed" structure $\left[1,\left[s^{*}\right]\right.$, [k]] for $k=1, \ldots, r$.

The approximate algorithm follows the below specified steps.

Step 0. Initialize the best found complete valid exposing structure $[b u, b S, b G]$ with value of $b H$ by the structure $\left[1,\left[s^{*}\right],[r]\right]$ where the minimal subscript $s^{*}$ is found by simple bisection process over range $[0 \ldots m]$.

Step 1. Repeat the following steps for $k=1 \ldots r$.

Step 2. Initialize the starting incomplete structure $[u, S, G]$ by valid incomplete exposing structure $\left[1,\left[s^{*}\right],[k]\right]$ where the subscript $s^{*}$ is found by simple bisection process over the range [sm..sM]. The limit $s m$ is set at zero for $k=1$ and it equals to $s^{*}$ at the next steps for the $k-1$. The limit $s M$ is specified using the value $b H$.

Step 3. Apply the procedure Complete on the structure $[u, S, G]$ and if a valid complete exposing structure is found and $H_{[u, S, G]}<$ $b H$ holds, then update the exposing structure $[b u, b S, b G]$ with value of $b H$ by the newly found structure.

\section{Computational study}

The main goal of this study is to verify the usefulness of suggested approximate algorithm for the min-max location problem with generalized system disutility. This problem represents the first step of the lexicographic optimization process [13]. It was found that this important first step is the most time-consuming part of the whole algorithm and therefore it is necessary to develop an effective solving method for this min-max problem. Within this paper, we try to answer the question whether the suggested algorithm based on radial formulation and exposing constraints considerably accelerates the solving process of the $p$-center problem. Therefore, we compare the basic radial approach based on the formulation (1) - (7) to the suggested method described in the previous section. The results are compared from the viewpoint of computational time and solution accuracy. All reported experiments were performed using the optimization software FICO Xpress 7.3 (64-bit, release 2012) for both studied approaches. The associated code was run on a PC equipped with the Intel ${ }^{\circledR}$ Core $^{\mathrm{TM}}$ i7 $2630 \mathrm{QM}$ processor with parameters: $2.0 \mathrm{GHz}$ and $8 \mathrm{~GB}$ RAM.

Particular approaches were tested on the pool of benchmarks obtained from the road network of the Slovak Republic. The instances are organized so that they correspond to the administrative organization of Slovakia. For each self-governing region (Bratislava - BA, Banska Bystrica - BB, Kosice - KE, Nitra - NR, Presov - PO, Trencin - TN, Trnava - TT and Zilina - ZA) all 
cities and villages were taken as possible service center locations and also as the user locations. Thus the number of possible service center locations $|I|$ is the same as the number of user locations
$|J|$ in all solved instances. The road network distance from a user located at $j$ to the center located at $i$ was taken as an individual user's disutility $d_{i j j^{\prime}}$ The value of parameter $p$ limiting the number

The results of numerical experiments for $r=3$ and the sets of coefficients $\boldsymbol{q}^{I}$ and $\boldsymbol{q}^{2}$.

Table 5

\begin{tabular}{|c|c|c|c|c|c|c|c|c|c|c|}
\hline \multirow{3}{*}{ Region } & \multirow{3}{*}{$|I|$} & \multirow{3}{*}{$p$} & \multicolumn{4}{|c|}{$q^{1}$} & \multicolumn{4}{|c|}{$q^{2}$} \\
\hline & & & \multicolumn{2}{|c|}{ RA_EC } & \multicolumn{2}{|c|}{ RA_EX } & \multicolumn{2}{|c|}{ RA_EC } & \multicolumn{2}{|c|}{ RA_EX } \\
\hline & & & $G^{*}$ & $\mathrm{CT}$ & $G^{*}$ & CT & $G^{*}$ & CT & $G^{*}$ & $\mathrm{CT}$ \\
\hline BA & 87 & 15 & 15.70 & 0.56 & 15.20 & 3600.44 & 12.85 & 0.51 & 14.50 & 3600.91 \\
\hline BA & 87 & 9 & 20.90 & 0.45 & 19.80 & 3601.11 & 17.45 & 0.48 & 17.00 & 3601.75 \\
\hline BB & 515 & 103 & 13.80 & 8.60 & 16.40 & 3603.01 & 11.40 & 9.00 & 16.30 & 3601.92 \\
\hline BB & 515 & 52 & 20.80 & 11.51 & 24.30 & 3602.16 & 16.90 & 11.97 & 21.20 & 3601.72 \\
\hline $\mathrm{KE}$ & 460 & 92 & 12.40 & 7.57 & 18.20 & 3601.86 & 9.70 & 7.91 & 13.95 & 3602.73 \\
\hline $\mathrm{KE}$ & 460 & 46 & 18.30 & 9.70 & 21.00 & 3607.53 & 15.15 & 9.84 & 19.95 & 3601.34 \\
\hline NR & 350 & 70 & 13.20 & 5.80 & 19.40 & 3609.43 & 11.10 & 7.10 & 16.95 & 3600.13 \\
\hline NR & 350 & 35 & 19.60 & 16.52 & 22.40 & 3606.02 & 16.30 & 12.40 & 20.95 & 3645.16 \\
\hline PO & 664 & 133 & 11.90 & 13.76 & 51.60 & 3765.59 & 9.95 & 14.71 & 45.30 & 3627.96 \\
\hline PO & 664 & 67 & 18.60 & 15.69 & 62.60 & 3622.00 & 15.30 & 17.32 & 54.80 & 3727.96 \\
\hline $\mathrm{TN}$ & 276 & 56 & 13.10 & 2.26 & 18.40 & 3621.14 & 10.55 & 2.31 & 17.15 & 3646.77 \\
\hline $\mathrm{TN}$ & 276 & 28 & 20.10 & 2.84 & 20.90 & 3604.75 & 16.05 & 2.86 & 18.40 & 3621.81 \\
\hline TT & 249 & 50 & 12.80 & 2.84 & 17.60 & 3843.03 & 10.40 & 3.84 & 15.20 & 3619.97 \\
\hline TT & 249 & 25 & 19.30 & 5.40 & 23.10 & 3622.38 & 16.15 & 5.63 & 19.85 & 3602.48 \\
\hline $\mathrm{ZA}$ & 315 & 63 & 15.20 & 2.78 & 15.30 & 3614.60 & 11.60 & 2.81 & 17.40 & 3606.02 \\
\hline $\mathrm{ZA}$ & 315 & 32 & 22.40 & 3.70 & 24.00 & 3631.00 & 18.20 & 3.78 & 23.30 & 3644.73 \\
\hline
\end{tabular}

The results of numerical experiments for $r=3$ and the sets of coefficients $q^{3}$ and $q^{4}$.

Table 6

\begin{tabular}{|c|c|c|c|c|c|c|c|c|c|c|}
\hline \multirow{3}{*}{ Region } & \multirow{3}{*}{$|I|$} & \multirow{3}{*}{$p$} & \multicolumn{4}{|c|}{$q^{3}$} & \multicolumn{4}{|c|}{$q^{4}$} \\
\hline & & & \multicolumn{2}{|c|}{ RA_EC } & \multicolumn{2}{|c|}{ RA_EX } & \multicolumn{2}{|c|}{ RA_EC } & \multicolumn{2}{|c|}{ RA_EX } \\
\hline & & & $G^{*}$ & $\mathrm{CT}$ & $G^{*}$ & $\mathrm{CT}$ & $G^{*}$ & CT & $G^{*}$ & $\mathrm{CT}$ \\
\hline BA & 87 & 15 & 23.30 & 0.37 & 21.80 & 3599.77 & 34.70 & 0.42 & 31.40 & 3600.95 \\
\hline BA & 87 & 9 & 30.10 & 0.47 & 29.00 & 3608.97 & 43.90 & 0.47 & 43.70 & 3603.82 \\
\hline BB & 515 & 103 & 20.20 & 7.66 & 24.90 & 3603.13 & 29.80 & 8.42 & 38.60 & 3601.73 \\
\hline BB & 515 & 52 & 31.20 & 11.11 & 35.40 & 3602.28 & 46.80 & 12.12 & 44.50 & 3624.90 \\
\hline $\mathrm{KE}$ & 460 & 92 & 19.60 & 7.63 & 29.40 & 3603.13 & 30.40 & 8.44 & 28.70 & 3641.84 \\
\hline $\mathrm{KE}$ & 460 & 46 & 26.70 & 9.03 & 29.70 & 3620.16 & 39.30 & 10.11 & 40.80 & 3751.00 \\
\hline NR & 350 & 70 & 18.80 & 6.36 & 26.50 & 3614.49 & 27.20 & 5.54 & 31.60 & 3690.81 \\
\hline NR & 350 & 35 & 28.40 & 16.49 & 31.10 & 3614.06 & 41.60 & 17.71 & 41.10 & 3627.44 \\
\hline $\mathrm{PO}$ & 664 & 133 & 17.10 & 11.33 & 68.30 & 3742.47 & 24.90 & 13.68 & 93.80 & 4054.29 \\
\hline PO & 664 & 67 & 27.40 & 17.05 & 83.10 & 3620.95 & 40.60 & 19.03 & 115.20 & 3614.31 \\
\hline $\mathrm{TN}$ & 276 & 56 & 19.90 & 2.20 & 20.00 & 3608.84 & 30.10 & 2.06 & 30.60 & 3618.53 \\
\hline $\mathrm{TN}$ & 276 & 28 & 30.90 & 2.81 & 29.40 & 3604.01 & 47.10 & 2.51 & 43.60 & 3616.31 \\
\hline TT & 249 & 50 & 19.20 & 3.70 & 25.40 & 3626.68 & 28.80 & 2.71 & 29.40 & 3620.81 \\
\hline TT & 249 & 25 & 27.70 & 5.13 & 27.60 & 3601.78 & 40.30 & 6.12 & 41.10 & 3610.05 \\
\hline $\mathrm{ZA}$ & 315 & 63 & 24.80 & 3.81 & 20.90 & 3609.09 & 37.20 & 2.70 & 32.50 & 3612.57 \\
\hline $\mathrm{ZA}$ & 315 & 32 & 33.60 & 3.48 & 35.60 & 3603.36 & 50.40 & 3.31 & 45.30 & 3610.83 \\
\hline
\end{tabular}


The results of numerical experiments for $r=5$ and the sets of coefficients $\boldsymbol{q}^{5}$ and $\boldsymbol{q}^{6}$.

Table 7

\begin{tabular}{|c|c|c|c|c|c|c|c|c|c|c|}
\hline \multirow{3}{*}{ Region } & \multirow{3}{*}{$|I|$} & \multirow{3}{*}{$p$} & \multicolumn{4}{|c|}{$q^{5}$} & \multicolumn{4}{|c|}{$q^{6}$} \\
\hline & & & \multicolumn{2}{|c|}{ RA_EC } & \multicolumn{2}{|c|}{ RA_EX } & \multicolumn{2}{|c|}{ RA_EC } & \multicolumn{2}{|c|}{ RA_EX } \\
\hline & & & $G^{*}$ & CT & $G^{*}$ & CT & $G^{*}$ & CT & $G^{*}$ & CT \\
\hline BA & 87 & 15 & 26.15 & 1.08 & 25.25 & 3600.07 & 47.20 & 0.92 & 39.30 & 3600.61 \\
\hline BA & 87 & 9 & 33.55 & 0.84 & 34.40 & 3601.53 & 50.80 & 0.84 & 54.10 & 3600.52 \\
\hline BB & 515 & 103 & 22.60 & 15.07 & 33.50 & 3602.90 & 34.60 & 18.81 & 59.30 & 3602.42 \\
\hline $\mathrm{BB}$ & 515 & 52 & 35.10 & 28.21 & 43.95 & 3603.12 & 57.60 & 26.35 & 54.10 & 3623.71 \\
\hline $\mathrm{KE}$ & 460 & 92 & 24.65 & 18.30 & 26.40 & 3602.82 & 36.80 & 18.75 & 35.70 & 3603.80 \\
\hline $\mathrm{KE}$ & 460 & 46 & 29.85 & 18.94 & 33.15 & 3601.38 & 52.40 & 17.44 & 49.60 & 3844.39 \\
\hline NR & 350 & 70 & 20.90 & 12.79 & 31.85 & 3623.97 & 31.40 & 14.43 & 35.10 & 3613.47 \\
\hline NR & 350 & 35 & 31.70 & 32.12 & 35.50 & 3614.09 & 56.40 & 33.04 & 70.00 & 3605.68 \\
\hline $\mathrm{PO}$ & 664 & 133 & 19.05 & 26.75 & 77.25 & 3633.24 & 28.80 & 25.29 & 111.20 & 3653.09 \\
\hline $\mathrm{PO}$ & 664 & 67 & 30.70 & 35.30 & 97.70 & 3626.94 & 54.80 & 44.60 & 143.40 & 3629.45 \\
\hline $\mathrm{TN}$ & 276 & 56 & 62.95 & 4.15 & 25.55 & 3642.50 & 88.50 & 4.84 & 35.50 & 3602.71 \\
\hline $\mathrm{TN}$ & 276 & 28 & 62.95 & 4.95 & 34.15 & 3605.82 & 88.50 & 5.23 & 53.00 & 3619.68 \\
\hline TT & 249 & 50 & 21.60 & 6.66 & 26.30 & 3636.78 & 37.00 & 6.86 & 35.50 & 3607.80 \\
\hline TT & 249 & 25 & 30.85 & 11.20 & 33.40 & 3603.63 & 46.60 & 14.40 & 49.10 & 3607.02 \\
\hline $\mathrm{ZA}$ & 315 & 63 & 44.45 & 5.02 & 27.10 & 3636.74 & 62.50 & 5.74 & 42.90 & 3611.83 \\
\hline $\mathrm{ZA}$ & 315 & 32 & 37.80 & 7.13 & 39.65 & 3624.17 & 58.80 & 7.22 & 54.10 & 3608.34 \\
\hline
\end{tabular}

of located service centers was set in such a way that the ratio of $|I|$ to $p$ equals 5 and 10 respectively. In the benchmarks, the generalized disutility perceived by any user sharing given location $j$ was defined by the sum of $r=3$ distances from the user's location to the three nearest located service centers. Particular disutility values are multiplied by the reduction coefficients $q_{k}$ for $k=1 \ldots r$ so that the biggest coefficient multiplies the smallest distance etc. The four triples $\boldsymbol{q}^{l}, \boldsymbol{q}^{2}, \boldsymbol{q}^{3}, \boldsymbol{q}^{4}$ of the reduction coefficients define the individual benchmarks and these symbols of the triples are used for distinguishing the results obtained by individual approaches applied on the benchmarks. The used triples were $\boldsymbol{q}^{I}=[1,0.2$, $0.1], \boldsymbol{q}^{2}=[1,0.1,0.05], \boldsymbol{q}^{3}=[1,0.5,0.2]$ and $\boldsymbol{q}^{4}=[1,0.8,0.5]$. The associated results of numerical experiments are reported in the following Table 5 and Table 6 where the basic radial exact approach based on the model (1) - (7) is denoted by RA_EX and the radial approach with exposing constraints is denoted by RA EC. The computational time in seconds is given in the columns denoted by CT and the symbol $G^{*}$ denotes the best found value of the generalized disutility, which corresponds to the maximal disutility perceived by the most exposed users of the designed public service system. Since our preliminary experiments showed that the used IP-solver needs unpredictable computational time when the middle-size integer programming problem with the min-max criterion is solved to optimality, we decided to test each method in the maximal time of one hour and we report the best achieved results at that time.

To make the comparison of presented approaches more precise, we enriched the pool of benchmarks in such a way that we increased the value of disutility contributing centers $r$ from 3 to 5 . Of course, we had to define new sets of reduction coefficients $q_{k}$. For this purpose we tested two different settings: $\boldsymbol{q}^{5}=[1,0.5$, $0.2,0.1,0.05]$ and $\boldsymbol{q}^{6}=[1,0.8,0.5,0.2,0.1]$. The results are reported in Table 7 which follows the same denotation as the previous tables.

The reported results indicate that the suggested algorithm based on exposing constraints gives more precise results in considerably shorter time in comparison to the exact method. We presume that the link-up constraints for the upper bound definition significantly spoil the convergence of the computational process based on the branch and bound principle. Therefore, we can conclude that our algorithm constitutes an effective solving tool for the min-max optimal public service system design problem with generalized system disutility.

The instances where our suggested algorithm lost in comparison to the prematurely terminated branch and bound approach as concerns the solution quality will become a topic of our future research.

\section{Conclusions}

The main goal of this study was to introduce and compare different approaches to the min-max optimal public service system design problem as the initial step of the lexicographic optimization process. Within this paper, the generalized system disutility was studied. The model of generalized disutility impacts the 
complexity of the problems which must be solved by used solving techniques. The consequence of the generalized disutility model is that the suitability of common approaches considerably changes when the min-max optimal public service system is designed. Based on reported results, the radial formulation of the problem with the min-max criterion does not hold for large instances because it proved to be extremely demanding as concerns the computational time and computer memory. Therefore, we have introduced and verified an advanced approximate algorithm for the min-max location problem based on radial formulation and exposing constraints. The results of experiments on real instances have confirmed its usefulness. Furthermore, presented method enables its simple implementation within common optimization environment instead of the necessity of developing special software tool. Thus, we can conclude that we have constructed a very useful solving tool for the middle-sized min-max optimal public service system design problem.

\section{Acknowledgement}

This work was supported by the research grants VEGA 1/0339/13 "Advanced microscopic modelling and complex data sources for designing spatially large public service systems“, APVV0760-11 "Designing of Fair Service Systems on Transportation Networks" and also by the project: University Science Park of the University of Zilina (ITMS: 26220220184) supported by the Research \& Development Operational Program funded by the ERDF.

\section{References}

[1] CURRENT, J., DASKIN, M., SCHILLING, D.: Discrete Network Location Models. In: Drezner, Z. (Ed.). Facility Location Applications and Theory, Berlin: Springer, pp. 81-118, 2002.

[2] INGOLFSSON, A., BUDGE, S., ERKUT, E.: Optimal Ambulance Location with Random Delays and Travel Times. Health Care Management Science, 11 (3), 262-274, 2008.

[3] JANOSIKOVA, L.: Emergency Medical Service Planning. Communications - Scientific Letters of the University of Zilina, 9 (2), 64-68, 2007.

[4] MARIANOV, V., SERRA, D.: Location Problems in the Public Sector. In: Drezner, Z. (Ed.): Facility location - Applications and theory, pp. 119-150, Berlin: Springer, 2002.

[5] AVELlA, P., SASSANO, A., VASILEV, I.: Computational Study of Large Scale p-median Problems. Mathematical Programming, 109, 89-114, 2007.

[6] KVET, M.: Computational Study of Radial Approach to Public Service System Design with Generalized Utility. Digital Technologies 2014 : Proc. of the $10^{\text {th }}$ Intern. IEEE Conference : Zilina, pp. 198-208, 2014.

[7] JANACEK, J., JANOSIKOVA, L.: Computability of the Emergency Service System Design Problem. Communications - Scientific Letters of the University of Zilina, ISSN 1335-4205, vol. 10, No. 2 (2008), pp. 5-9.

[8] JANACEK, J.: Radial Approach to the Emergency Public Service System Design with Generalized System Utility. Intern. J. of Applied Mathematics and Informatics, 8, 7-14, 2014.

[9] BERTSIMAS, D., FARIAS, V. F., TRICHAKIS, N.: The Price of Fairness. Oper. Res., 59, 17-31, 2011.

[10] BUZNA, L., KOHANI, M., JANACEK, J.: Proportionally Fairer Public Service Systems Design. Communications - Scientific Letters of the University of Zilina, ISSN 1335-4205, vol. 15, No. 1, 2013, pp. 14-18.

[11] MARSH, M., SCHILLING, D.: Equity Measurement in Facility Location Analysis. European J. of Operational Research, 74, 1-17, 1994.

[12] NASH, J.: The Bargaining Problem. Econometrica, 18 (2), 155-162, 1950.

[13] OGRYCZAK, W., SLIWINSKI, T.: On Direct Methods for Lexicographic Min-Max Optimization. In: Gavrilova M. et al. (Eds.): ICCSA 2006, LNCS 3982, (pp. 802-811). Berlin: Heidelberg: Springer, 2006.

[14] GARCIA, S., LABBE, M., MARIN, A.: Solving Large p-median Problems with a Radius Formulation. INFORMS J. on Computing, 23 (4), 546-556, 2011.

[15] JANACEK, J.: Approximate Covering Models of Location Problems. Lecture Notes in Management Science: Proc. of $1^{\text {st }}$ Intern. Conference ICAOR '08, vol. 1, Sept. 2008, Yerevan, pp. 53-61, 2008.

[16] JANACEK, J., KVET, M.: Relevant Network Distances for Approximate Approach to the p-median Problem. Operations Research Proceedings 2012: Selected Papers of the Intern. Conference of the German operations research society (GOR), September 2012, Leibniz: Univesitat Hannover: Springer, pp. 123-128, 2014.

[17] SNYDER, L. V., DASKIN, M. S.: Reliability Models for Facility Location; The Expected Failure Cost Case. Transport Science, 39 (3), 400-416, 2005. 